\title{
A NEW APPROACH TO THE EVALUATION OF LOCAL MUSCULAR LOAD WHILE TYPING ON A KEYBOARD
}

\author{
Anna Schlenker ${ }^{1,2}$, Tomáš Tichý ${ }^{3}$ \\ ${ }^{1}$ Institute of Hygiene and Epidemiology, First Faculty of Medicine, Charles University and General University Hospital in Prague, Prague, Czech \\ Republic \\ ${ }^{2}$ Department of Biomedical Informatics, Faculty of Biomedical Engineering, Czech Technical University in Prague, Kladno, Czech Republic \\ ${ }^{3}$ National Institute of Public Health, Prague, Czech Republic
}

\begin{abstract}
SUMMARY
Objective: The aim of this study was to assess the contribution of using keystroke dynamics (KD) in combination with integrated electromyography (iEMG) for the objective evaluation of local muscular load of hands and forearms while typing on a computer keyboard and to compare it with results of the commonly used method.

Method: Study was performed on 12 subjects. Data were collected using our own application for capturing KD data and using EMG Holter for detecting electromyographic potentials to determine local muscular load.

Results: The results of our study revealed that currently used methods for assessment of the workload while typing on a computer keyboard are not entirely accurate. In particular, the real total number of keystrokes performed during processing of a text is significantly higher than the count of characters the text is consisting of. In addition to this count, also the so-called invisible keys, keyboard shortcuts, and especially corrections in the typed text must be taken into consideration.

Conclusions: The results indicated that all probands in our study exceeded the valid hygienic limits for the total amount of the small repetitive movements of the hands and forearms and the total amount of the keyboard typing movements. Most of the probands in our study also exceeded the valid hygienic limit for the highest average time-weighted value of the percent maximum voluntary contraction (\%MVC). This implies that the keystroke dynamics method has a great potential to increase the accuracy of evaluation of local muscular load when using the keyboard and thus to improve the existing methodology for investigation of occupational diseases resulting from overload while working on the computer.
\end{abstract}

Key words: integrated electromyography, keystroke dynamics, local muscular load evaluation, hygienic limits, prevention of repetitive strain injury

Address for correspondence: A. Schlenker, Institute of Hygiene and Epidemiology, First Faculty of Medicine, Charles University and General University Hospital in Prague, Studničkova 7, 12800 Prague 2, Czech Republic. E-mail: schlenker.anna@gmail.com

https://doi.org/10.21101/cejph.a5105

\section{INTRODUCTION}

In the Czech Republic, the incidence of occupational diseases due to the long-term excessive unilateral load of the upper extremities is increasing during recent years. The frequent cause is the prolonged excessive burden on small muscle groups of the forearm and hand, so-called local muscular load. This burden is evaluated primarily using integrated electromyography (iEMG). Objectification of local muscular load is performed mainly for work associated with high numbers of repeated unilateral movements (in forced pace of work, assembly work, minor installation work demanding on precision, typing, etc.). Optionally, it is at work, in which large muscular forces are expended (handling heavy loads, applying high finger forces in assembly tasks, work associated with unfavourable working postures of the upper limbs, etc.).

The evaluation of load with this method is based on the limits set by Government Decree no. 361/2007 Coll., as amended, establishing conditions of health protection at work. We evaluate the average time-weighted value of the percent maximum voluntary contraction (\%MVC), the number of expended large muscle forces in the range of 55-70\% MVC, incidence of the over-limit muscle forces above $70 \% \mathrm{MVC}$, and the whole shift number of unilateral repetitive movements of hands and forearms with regard to expended forces. In terms of working with a computer, it is the excessive number of repeated unilateral movements with regard to expended muscle strength during typing and using a mouse, which represents one of the potential risk factors for an occupational/work related disease due to overload.

The evaluation of movements during the typing is currently performed in three ways: the method of in-situ observation takes place several times over a limited period of time in order to determine the movements per minute of the hands and forearms. The worker is under the direct supervision of the evaluating person. It can stress him/her, he or she can work at a different speed than usual, and may make more mistakes.

Analysis of the occupational activities from video recordings is carried out in a similar way as the method of in-situ observation, i.e. few times over a limited period of time to determine the number of movements per minute of the hands and forearms. During video recording, the evaluated worker can be exposed to the same level of stress as in the first method. 
Evaluation of the amount of written text has the disadvantage that the final text does not include complete information concerning the total amount of work done on the computer keyboard. Relevant examples are typing errors, i.e. characters (whole words, sentences or paragraphs) that were written during the work and subsequently deleted. Furthermore, this includes the "in the text invisible" keystrokes such as "Shift" or "Caps Lock" when writing capital letters, pressing "Delete" or "BackSpace" when correcting typos, pressing "Tab" or "Enter" when going through web forms, etc. Additionally, it is a combination of keystrokes, such as " $\mathrm{Ctrl}$ $+\mathrm{A}$ " to select text, "Ctrl + C" to copy or "Ctrl + X" to cut the text and then "Ctrl $+\mathrm{V}$ " to paste it. These keystrokes are not seen in the final text, and quite often, they are a very abundant component when creating different types of documents.

For the above-mentioned reasons, none of those standard methods can objectively determine the actual number of movements during typing or using a mouse. Therefore, improvement of the existing methodology for measuring and evaluating local muscular load while working on a computer is needed, especially for the work which is characterised by a high frequency of entering data into the computer via keyboard (e.g. accountant, clerk, secretary, etc.). In an effort to improve objectivity and accuracy of determination of the number of movements while working on the computer and of the evaluation of the local muscular load, we decided to combine iEMG with keystroke dynamics (KD) method. Our study was designed and planned with the aim to evaluate the potential contribution of this modification.

\section{MATERIALS AND METHODS}

All probands were typing the same text. They were either copying it from a computer screen in front of them or from the paper on an easel (according to current possibilities and user preferences). Users also had the opportunity to try different styles of typing/ keyboarding (according to technical possibilities available). The evaluating person observes a worker, checks the whole process of measuring and makes notes about specific characteristics of the user's work with a computer.

The text chosen for typing should not be too long, too complicated, dealing with a specific topic, but it should contain special characters and numbers. The specific text taken from the pages of the Czech news server consisted of the title and eight paragraphs containing 307 words and 1,735 characters (2,033 characters including spaces) (1). Probands had the opportunity to read the text in advance.

Measurement took 30 minutes. If the proband was typing fast, the text might have been written repeatedly during the interval of 30 minutes.

\section{Data Collection}

The study group contained 12 probands ( 7 females, 5 males), the average age was 39.6 years (median 34). The group was divided into two subgroups. Subgroup 1 comprised people occupationally using typing/keyboarding (typing with 10 fingers) and subgroup 2 people well typing on a keyboard, but not occupationally, i.e. they did not type with all 10 fingers but used both hands, more than two fingers and typed relatively quickly without many typos. Both subgroups of people used the computer for their everyday work. The measured dataset was checked for factual and formal errors. The dataset contained no errors so no record was excluded from further processing.

\section{Keystroke Dynamics}

Keystroke dynamics is a behavioural biometric characteristic based on monitoring user behaviour while interacting with the keyboard. We measure time intervals between individual keystrokes and duration of individual keystrokes. By combining these features, a timing vector is formed, which is able to clearly determine how many keystrokes, in fact, the user pressed while interacting with the keyboard and how long period of time he/ she spent on typing/keyboarding.

For KD measurement we use our own specialised software application, which was upgraded for this measurement from previous versions $(2-4)$.

\section{Integrated Electromyography}

Integration is a mathematical process that calculates the area circumscribed by the curve. For the integration of electromyography (EMG) signals, a full-wave rectifier and an electronic integrator are used. Integrated electromyogram represents the total muscle activity and is a function of the amplitude, duration and frequency of the course of each EMG potential. For measurements, a portable eight-channel polygraph was used to record physiological parameters, EMG Holter with 4 EMG modules. EMG module is used to track muscle activity by measuring and recording electrical potentials accompanying muscle activity. EMG potentials are scanned by special surface electrodes. The EMG Holter from GETA Centrum Company (5) was used for local muscular load measuring.

\section{RESULTS}

Table 1 provides the total number of keystrokes during the whole measured period (30 minutes), the total number of "Space" and "Return" keys which are usually well visible in the final text and also the total amount of the "invisible" keystrokes which were used by probands in our experiment. These "invisible" keystrokes are mainly the keys used for correcting the misprints like "BackSpace" and/or "Delete" keys, keys used for typing capital letters like "LeftShift", "RightShift" and/or "CapsLock" and keys used for movements in the text, mainly the arrow keys (Left, Right, Up and Down). We measured also the number of other "invisible" keystrokes which occurred in our data (e.g. Escape, LeftCtrl, Home, End, LeftMenu, RightMenu, etc.), but their representation was not significant in our set (these occurred only in two of our probands and the total numbers was under 10).

Table 1 also shows that the most common way for correcting typos in our experiment is using "BackSpace" key and only a half of our group used "Delete" key and in a much lesser extent. We can also see that the most common way for typing capital letters is using "Shift" key rather than using "CapsLock" key. Interestingly, one of the users never used "LeftShift" key during typing the 


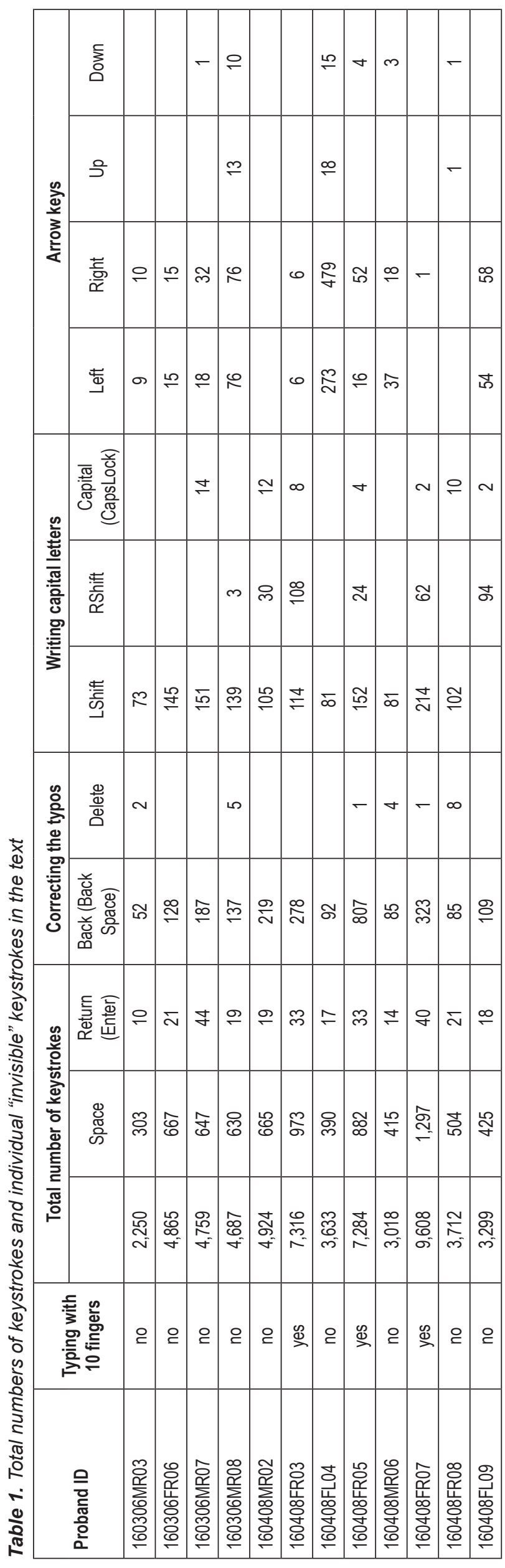

text, we can only guess that the reason could be, that this person is left-handed. But this assumption cannot be confirmed by the second left-handed user in our dataset who on the contrary used only "RightShift" key. Also, another proband used "LeftShift" and "RightShift" keys in the same ratio. Again we can only guess that this is because of the correct typing style with all 10 fingers. Using "CapsLock" key for typing capital letters occurred in 7 out of 12 users but for each of them only to a small extent. Motion in the text using arrow keys in the text is very individual, Table 1 shows one user who used them very often, but also one person who never used them and two persons who used them only few times (1-2 occurrence).

In Table 2 we introduce new variables, shortcuts and formulas for calculated values shown in the following tables. The formulas are applicable in cases where the user does not use mouse for marking parts of the text before deleting it or for moving in the text and even if he does, then only 1 or 2 times during the writing for marking one or two words in the text). In formulas for counting the net total amount of keystrokes without "invisible" keys, it is necessary to explain that we deduct also the double total number of BD (the sum of "Delete" and "BackSpace" keys). The reason is that if the user uses "Delete" or "BackSpace" key, he/she uses it usually for deleting some character in the text, so we assume, that the whole text will be shorter from these characters.

Table 3 shows the total numbers of individual groups of keystrokes for each user.

For the correct evaluation of workload while typing on a computer keyboard we also need to count the movements per minute of hands and forearms and average values of $\% \mathrm{MVC}$. The results are shown in Table 4 . We counted the movements per minute from a total number of keystrokes (TK) and from a net total number of keystrokes (NT). The average values of \%MVC are measured directly by the electromyography software.

More interesting is the comparison with currently valid legislation. Measured average values of $\% \mathrm{MVC}$ and the total movements per minute of the hands and forearms are generally higher than the current valid hygienic limits (3\% MVC and 110 movements per minute or $6 \% \mathrm{MVC}$ and 60 movements per minute for typing on a computer keyboard).

Figure 1 represents the movements per minute of the hands and forearms and valid hygienic limits for the total amount of small repetitive movements of the hands and forearms (110 movements per minute, solid line in the graph) and the total amount of keyboard typing movements (60 movements per minute, dashed line in the graph). The square marks in the graph represent the movements per minute of the hands and forearms counted from the net total number of keystrokes (NT) and the circular marks represent the movements per minute of the hands and forearms counted from the total amount of keystrokes sensed during typing the text (TK). Figure 1 clearly indicates that all users in our study exceeded valid hygienic limits.

Figure 2 represents the average time-weighted \%MVC value of flexors and extensors of the hands and forearms and valid hygienic limits for $\% \mathrm{MVC}$.

Figure 2 clearly indicates that the highest average values of $\% \mathrm{MVC}$ were noted in extensor muscle groups and for probands who type with all 10 fingers. Most of the probands in our study exceeded valid hygienic limits in some cases more than three times (the values exceeded 20\% MVC). 
Table 2. Description of shortcuts, variables and formulas used for data evaluation

\begin{tabular}{|l|l|l|l|}
\hline Variable & Shortcut & Description & Formula \\
\hline TK & Total keystrokes & The total amount of keystrokes sensed during typing the text & \\
\hline TW & Total without spaces & The total amount of keystrokes without "Space" and "Return" keys & \\
\hline BD & BackSpace Delete & $\begin{array}{l}\text { The sum of keystrokes used for correcting typos (the sum of "BackSpace" } \\
\text { and "Delete" key). }\end{array}$ & \\
\hline CL & Capital letters & $\begin{array}{l}\text { The sum of keystrokes used for writing capital letters, namely "LeftShift", } \\
\text { "RightShift" and/or "CapsLock" key. }\end{array}$ & \\
\hline AK & Arrow keys & $\begin{array}{l}\text { The total amount of movements in the text using the arrow keys "Up", } \\
\text { "Down", "Left" and "Right". }\end{array}$ & $\mathrm{NT}=\mathrm{TK}-\left(2^{*} \mathrm{BD}+\mathrm{CL}+\mathrm{AK}\right)$ \\
\hline NT & Net total & $\begin{array}{l}\text { The total number of keystrokes without "invisible" keys and deleted } \\
\text { characters. }\end{array}$ & $\begin{array}{l}\text { The total amount of keystrokes without "invisible" keys, deleted characters } \\
\text { and without spaces. }\end{array}$ \\
\hline NW & Net total without spaces $-\left(2^{*} \mathrm{BD}+\mathrm{CL}+\mathrm{AK}\right)$ \\
\hline
\end{tabular}

Table 3. Total numbers of the groups of keystrokes

\begin{tabular}{|l|c|c|c|c|c|c|c|c|c|}
\hline Proband ID & TK & Space & Return & TW & BD & CL & AK & NT & NW \\
\hline 160306MR03 & 2,250 & 303 & 10 & 1,937 & 54 & 73 & 19 & 2,050 & 1,737 \\
\hline 160306FR06 & 4,865 & 667 & 21 & 4,177 & 128 & 145 & 30 & 4,434 & 3,746 \\
\hline 160306MR07 & 4,759 & 647 & 44 & 4,068 & 187 & 165 & 51 & 4,169 & 3,478 \\
\hline 160306MR08 & 4,687 & 630 & 19 & 4,038 & 142 & 142 & 175 & 4,086 & 3,437 \\
\hline 160408MR02 & 4,924 & 665 & 19 & 4,240 & 219 & 147 & 0 & 4,339 & 3,655 \\
\hline 160408FR03 & 7,316 & 973 & 33 & 6,310 & 278 & 230 & 12 & 6,518 & 5,512 \\
\hline 160408FL04 & 3,633 & 390 & 17 & 3,226 & 92 & 81 & 785 & 2,583 & 2,176 \\
\hline 160408FR05 & 7,284 & 882 & 33 & 6,369 & 808 & 180 & 72 & 5,416 & 4,501 \\
\hline 160408MR06 & 3,018 & 415 & 14 & 2,589 & 89 & 81 & 58 & 2,701 & 2,272 \\
\hline 160408FR07 & 9,608 & 1,297 & 40 & 8,271 & 324 & 278 & 1 & 8,681 & 7,344 \\
\hline 160408FR08 & 3,712 & 504 & 21 & 3,187 & 93 & 112 & 2 & 3,412 & 2,887 \\
\hline 160408FL09 & 3,299 & 425 & 18 & 2,856 & 109 & 96 & 112 & 2,873 & 2,430 \\
\hline
\end{tabular}

Table 4. Movements per minute of hands and forearms counted from total keystrokes (TK) and net total (NT) variables and measured average values of percent maximum voluntary contraction (\%MVC) for right and left hand and forearm flexors and extensors

\begin{tabular}{|c|c|c|c|c|c|c|c|c|}
\hline \multirow[t]{2}{*}{ Proband ID } & \multicolumn{2}{|c|}{ Total number of keystrokes } & \multicolumn{2}{|c|}{$\begin{array}{l}\text { The movements per minute } \\
\text { of the hands and forearms }\end{array}$} & \multicolumn{2}{|c|}{$\begin{array}{l}\text { Average values of \%MVC } \\
\text { for right hand and forearm }\end{array}$} & \multicolumn{2}{|c|}{$\begin{array}{l}\text { Average values of \%MVC } \\
\text { for left hand and forearm }\end{array}$} \\
\hline & TK & NT & from TK & from NT & flexors & extensors & flexors & extensors \\
\hline 160306MR03 & 2,250 & 1,737 & 150 & 116 & 2.94 & 3.07 & 6.28 & 3.59 \\
\hline 160306FR06 & 4,865 & 3,746 & 324 & 250 & 11.37 & 6.73 & 9.30 & 14.08 \\
\hline 160306MR07 & 4,759 & 3,478 & 317 & 232 & 16.82 & 10.26 & 9.83 & 5.11 \\
\hline 160306MR08 & 4,687 & 3,437 & 312 & 229 & 12.68 & 21.91 & 11.84 & 9.52 \\
\hline 160408MR02 & 4,924 & 3,655 & 328 & 244 & 3.90 & 6.07 & 3.24 & 6.10 \\
\hline 160408FR03 & 7,316 & 5,512 & 488 & 367 & 3.70 & 20.33 & 4.17 & 24.44 \\
\hline 160408FL04 & 3,633 & 2,176 & 242 & 145 & 4.53 & 21.27 & 4.54 & 23.79 \\
\hline 160408FR05 & 7,284 & 4,501 & 486 & 300 & 15.89 & 17.04 & 14.54 & 26.13 \\
\hline 160408MR06 & 3,018 & 2,272 & 201 & 151 & 2.40 & 9.36 & 3.67 & 5.20 \\
\hline 160408FR07 & 9,608 & 7,344 & 641 & 490 & 11.16 & 16.68 & 5.55 & 20.23 \\
\hline 160408FR08 & 3,712 & 2,887 & 247 & 192 & 5.66 & 6.46 & 5.48 & 10.70 \\
\hline 160408FL09 & 3,299 & 2,430 & 220 & 162 & 6.54 & 10.95 & 7.51 & 8.69 \\
\hline
\end{tabular}




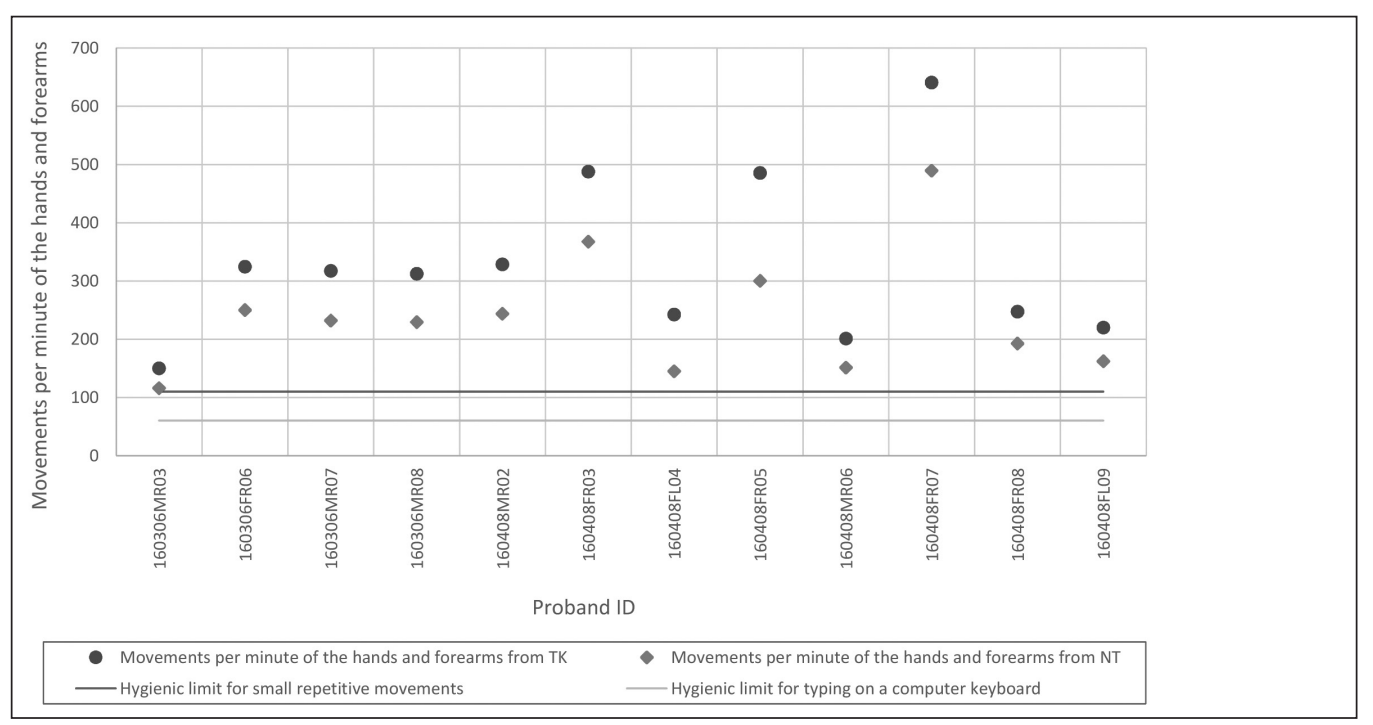

Fig. 1. Representation of movements per minute of the hands and forearms from all motions/keystrokes and from in the text visible keystrokes only.

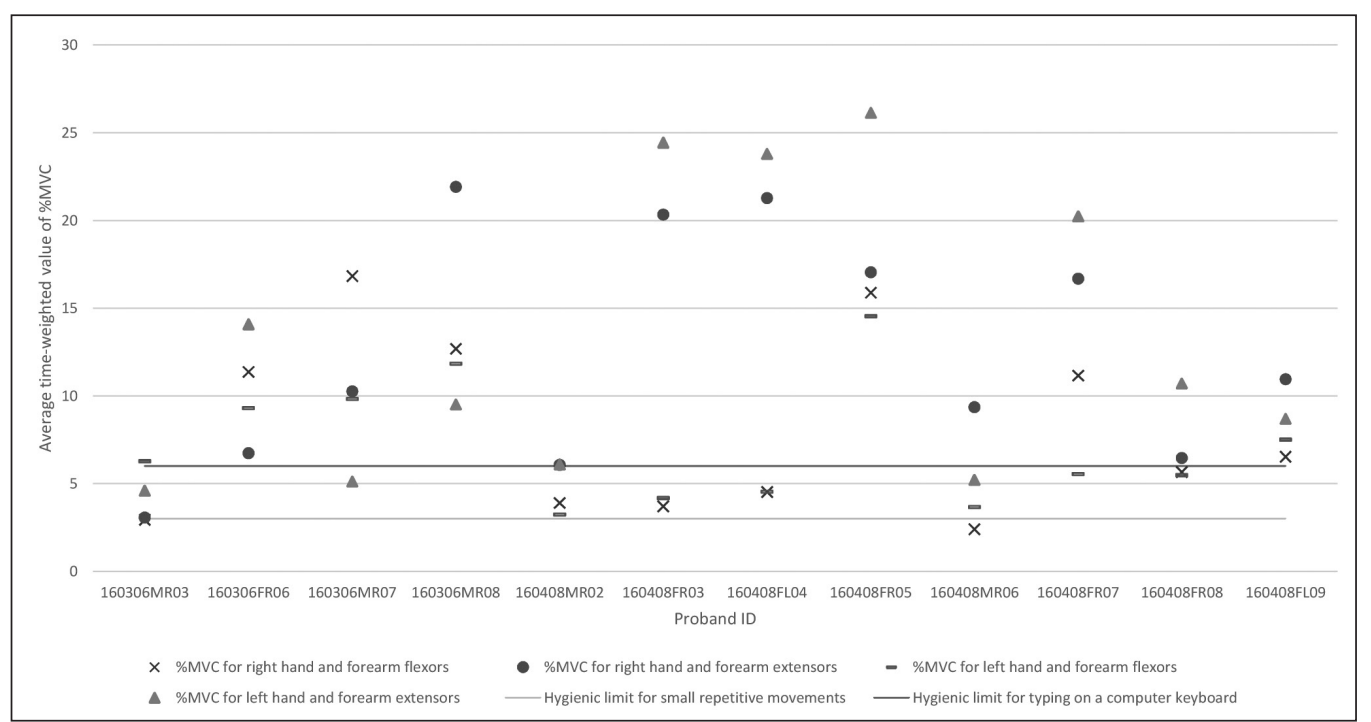

Fig. 2. Representation of the average time-weighted value of \%MVC (maximum voluntary contraction) of the flexors and extensors of the hands and forearms and the valid hygienic limits.

$3 \% \mathrm{MVC}$, dashed line in the graph, $6 \% \mathrm{MVC}$, the solid line in the graph. Square marks in the graph represent the average values of \%MVC for flexors of the right hand and forearm, the circular marks for extensors of the right hand and forearm. Triangular marks in the graph represent the average values of \%MVC for flexors of the left hand and forearm and diamond marks for extensors of the left hand and forearm.

The results of our study revealed that currently used methods for objective workload evaluation while typing on a computer keyboard are not entirely accurate. In particular, amounts of the written text evaluated according to the number of characters written is significantly higher because of the use of so-called invisible keys, keyboard shortcuts, and especially due to corrections in the typed text. This significant difference is seen even when the users do not use mouse when typing the text or at least use it only in a very limited way.

When comparing our measured results with valid hygienic limits, the limits on the number of key presses (the number of movements) per minute and also the average time-weighted value of \%MVC are exceeded in almost all the aforementioned cases.

\section{DISCUSSION}

The results of our study show that currently used methods for evaluation of workload while working with the computer keyboard are not objective. Evaluation of the number of repeated unilateral movements per minute by on-site observation or video analysis cannot capture all tiny movements of the fingers when typing on the keyboard. As we expected, the total number of characters (including spaces) in the final text calculated by a text editor is not objective basis for determining the number of keystrokes.

Due to the innovative idea of KD and iEMG simultaneous usage to evaluate local muscular load at work associated with use of the keyboard, the results of our study cannot be compared with other publications. Especially in Czech legislation, there is 
no similar idea covered. Integrated electromyography (iEMG) in combination with Keystroke dynamics (KD) is used, for example, in order to increase access security to specific computer applications and/or information systems (6). By monitoring a larger set of probands we can get behavioural characteristics of the clerks, which could allow us to create a higher-level user authentication method. In this method can be included, e.g. how the user uses invisible characters like Shift, CapsLock, etc. These characteristics of the use of "invisible" characters could supplement the results of simple keystroke dynamics and could be used in cases of password recovery, after repeated attempts to enter the password, etc.

Our method has proved in practice to be simple to use and not disturbing or limiting the worker during the work. It was also found that for all evaluated probands the respective hygienic limits were exceeded for the total number of repeated unilateral movements of hands and forearms with respect to expended $\% \mathrm{MVC}$. This raises the question whether the hygienic limits in Czech legislation are set up correctly and whether they respect the current situation when working on the computer, which changed markedly compared to the previous periods, and especially typing is associated with higher amounts of invisible keystrokes and with forced working postures of the wrist. Due to our limited dataset we cannot confirm or deny this hypothesis completely, so we are planning to continue with this issue on a much larger scale including both keystroke dynamics and mouse dynamics evaluation.

It should be mentioned that the measurement was carried out in the computer lab model conditions under supervision of the evaluating person for a limited time. These conditions can play an essential role in relation to the measured values of both keystrokes dynamics and expended forces. We assume that the results of measurements in practice could be slightly different.

The disadvantage of applied KD method in relation to the local muscular load is currently impossible recognition between the right and left hand while typing. We also plan to deal with this imperfection in the future.

Another important part of the local muscular load evaluation is during working with the computer mouse, especially in occupations like graphic designers. Also, the vast majority of workers use the mouse and computer keyboard in varying proportions while working with the computer. Therefore, we are planning to enrich our method with the scanning of mouse movements.

It should be noted that the aim of our study was not assessing the workplace ergonomics, working posture of hands and forearms or workload when working with a computer mouse. We focused exclusively on just one of the important factors, namely the total number of keystrokes.

\section{CONCLUSION}

In addition to a visual assessment of working postures in evaluation of the local muscular load while typing on a computer, two different techniques were used, namely the measurement of the local muscular load on the forearm using iEMG and KD measurement using specialised software.
The aim of our study was to verify simultaneous use of KD and iEMG methods for more accurate evaluation of workload while typing on a computer keyboard, particularly with regard to the repeated unilateral movements of small muscles of the hand and fingers during the work.

It was proven that the total amount of written text (and a total number of individual keystrokes), which was evaluated according to the number of characters written, is significantly higher due to the use of so-called "invisible" keystrokes, keyboard shortcuts, and especially due to corrections in the written text.

A study offers a possible way for practical use of this combination of methods, e.g. in the context of objectification of the workload (e.g. clerks, secretaries, accountants) or as part of an inquiry occurrence of occupational/work related diseases from the excessive unilateral load (carpal tunnel syndrome, lateral epicondylitis, etc.).

For the full implementation of this method, it is needed to explore the method widely and make some improvements. But the study results clearly demonstrate that current $\mathrm{KD}$ and $\mathrm{iEMG}$ use for these purposes will be possible.

Keystroke dynamics method has a great potential to improve the existing methodology for investigation of occupational diseases resulting from overloading while working on the computer.

\section{Acknowledgements}

We would like to thank Jana Hlávková for her valuable comments on our work; Michal Reimer for help with programming and countless small but significant software changes; Milan Šárek and Milan Tuček for comments that greatly improved the manuscript; and Anna Holubová for proofreading. We thank all probands who participated in our study. This research was partially supported by the PROGRESS Q25, Charles University, Prague.

\section{REFERENCES}

1. Bůnová M, Váchal A. The unique tram called "Evička" carries passengers, you meet it on three lines. iDNES.cz [Internet]. Mafra [cited 2016 Feb 5]. Available from: http://praha.idnes.cz/tramvaj-evicka-vozi-cestujici-dk8-/ praha-zpravy.aspx?c=A160205 120353 praha-zpravy nub. (In Czech.)

2. Reimer M. Identity authentication based on continuous keystroke dynamics [dissertation]. Kladno: České vysoké učení technické; 2015. (In Czech.)

3. Schlenker A, Reimer M. Big Data in hospital information systems in the terms of security. In: Svačina Š, Zvárová J, editors. Semantic interoperability in biomedicine and healthcare. Prague: Charles University; 2015. p. 39-41.

4. Schlenker A, Bohunčák A. Keystroke dynamics for security enhancement in hospital information systems. Int J Biomed Healthc. 2015;3(1):41-4.

5. EMG Holter. Physiology of work [Internet]. GETA Centrum; 2015 [cited 2017 Feb 16]. Available from: http://fyziologie.getacentrum.cz/ emg-holter/. (In Czech.)

6. Venugopalan S, Juefei-Xu F, Cowley B, Savvides M. Electromyograph and keystroke dynamics for spoof-resistant biometric authentication. In: 2015 IEEE Conference on Computer Vision and Pattern Recognition Workshops (CVPRW 2015); 2015 Jun 7-12; Boston, MA, USA. Curran Associates; 2016. p. 495-504. 